\title{
Experimental Passive Flutter Mitigation Using a Linear Tuned Vibrations Absorber
}

\author{
E. Verstraelen, G. Habib, G. Kerschen, G. Dimitriadis \\ Department of Aerospace and Mechanical Engineering, University of Liège, Belgium
}

\begin{abstract}
The current drive for increased efficiency in aeronautic structures such as aircraft, wind turbine blades and helicopter blades often leads to weight reduction. A consequence of this tendency can be increased flexibility, which in turn can lead to unfavourable aeroelastic phenomena involving large amplitude oscillations and nonlinear effects such as geometric hardening and stall flutter. Vibration mitigation is one of the approaches currently under study for avoiding these phenomena.

In the present work, passive vibration mitigation is applied to an experimental aeroelastic system by means of a linear tuned vibration absorber. The aeroelastic apparatus is a pitch and flap wing that features a continuously hardening restoring torque in pitch and a linear one in flap. Extensive analysis of the system with and without absorber at subcritical and supercritical airspeeds showed an improvement in flutter speed around 34\%, a suppression of a jump due to stall flutter, and a reduction in LCO amplitude.

Mathematical modelling of the experimental system showed that optimal flutter delay can be obtained when two of the system modes flutter simultaneously. However, the absorber quickly loses effectiveness as it is detuned. The wind tunnel measurements showed that the tested absorbers were much slower to lose effectiveness than those of the mathematical predictions.
\end{abstract}

Keywords: Wind Tunnel Testing, Nonlinear Aeroelasticity, Stall Flutter, Internal Resonance, Bifurcations.

\section{INTRODUCTION}

As aircraft become lighter and are pushed further in their flight envelope for performance reasons, they are more prone to undergo unfavourable aeroelastic oscillations due to flutter, a very dangerous mechanism occurring because of the coalescence of two vibration modes subjected to an airflow.

Common methods to avoid these oscillations include reducing the flight envelope, stiffening the structure, active control and increasing structural damping. The employment of a linear tuned vibration absorber (LTVA), also known as tuned mass damper (TMD), is an alternative vibration control approach that has not yet been fully explored in the aeroelastic literature.

The LTVA consists of a mass-spring-damper assembly attached to the primary system to be controlled. The natural frequency of the LTVA is tuned in accordance to the main resonant frequency of the host system, causing an effective transfer of energy between the two subsystems. The relatively high damping of the absorber reduces significantly the oscillation amplitude. LTVAs are widely used in civil engineering applications, such as tall buildings ${ }^{[1]}{ }^{[2]}$ and long span bridges ${ }^{[3]}$, to avoid vortex induced vibrations, galloping oscillations or human-induced vibrations ${ }^{[4]}$. However, in classical flutter, the loss of stability is due to two modes whose frequencies vary with the airspeed. The variation of the resonant frequencies undermines the 1:1 resonance, crucial for the correct operation of the LTVA. The design of an effective LTVA for increasing the flutter speed in aeroelastic system is thus more challenging. Analytical and numerical works showed that the LTVA can be successfully implemented for the suppression of flutter vibrations in long span 
bridges ${ }^{[5-8]}$. A few pioneering studies ${ }^{[9,10]}$ have illustrated that the same concept can be applied to aircraft wings. However, to the authors' knowledge, there is a lack of experimental work demonstrating the effectiveness of LTVAs for flutter suppression in the literature.

In this study we attach a LTVA to a nonlinear pitch and flap wing (NLPFW) in order to delay the flutter onset speed. The study is conducted in three steps. Firstly, the aeroelastic system's behaviour is extensively investigated numerically and experimentally in the wind-tunnel. Secondly, numerical and experimental LTVAs are designed. Finally, the efficiency of these absorbers is assessed and tuning rules are derived.

\section{PRIMARY SYSTEM}

\subsection{Experimental setup}

The experimental apparatus, installed in the large low-speed wind tunnel of the University of Liège, is designed to achieve very low linear damping $(\approx 0.3 \%$ at wind-off conditions) and flutter at an airspeed close to $12 \mathrm{~m} / \mathrm{s}$. To achieve such low structural damping, the apparatus does not use any bearings or rotational springs. The pitch and flap restoring torques are provided by a specially designed leaf spring and a nonlinear clamp assembly. The complete NLPFW is shown in Fig. 1. It is a stiff thin rectangular unswept

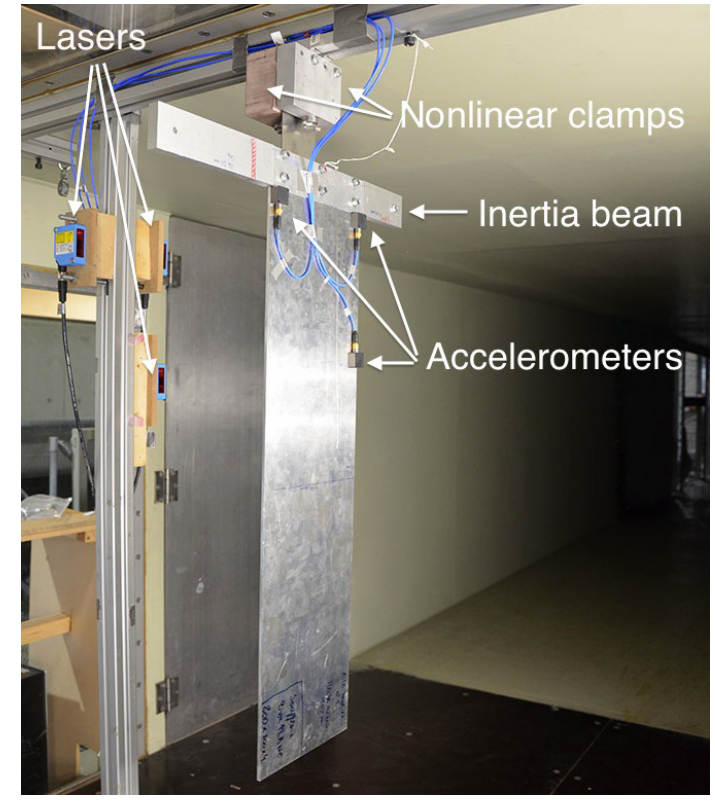

(a) Photo of the setup in the wind tunnel

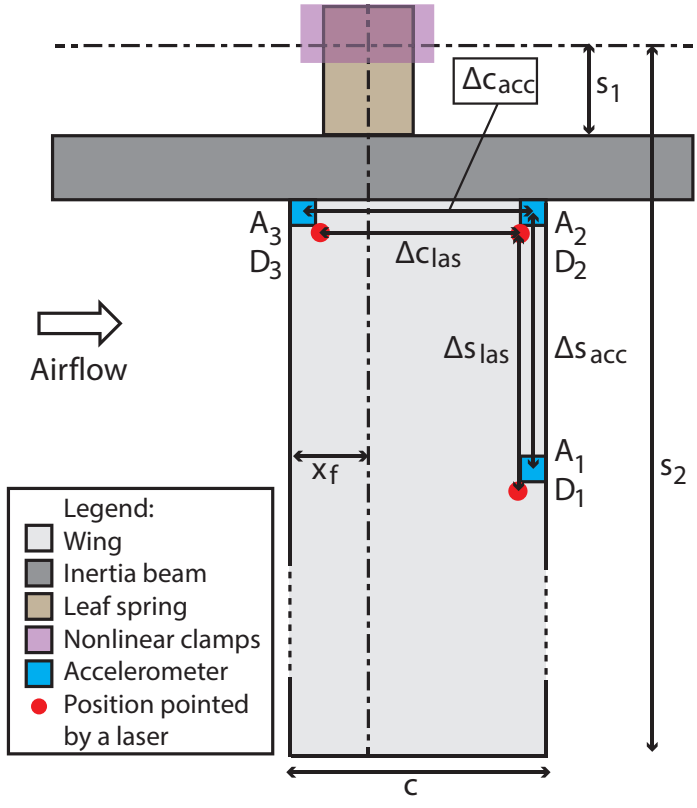

(b) Diagram showing transducer locations and major components of the NLPFW

Figure 1: Experimental setup showing wing, support and transducers

flat plate with span $s=800 \mathrm{~mm}$, chord $c=200 \mathrm{~mm}$, thickness $t=4 \mathrm{~mm}$ and an aspect ratio of 4 . It is hinged at its root at $0.3 c$ from the leading edge. It features two rigid degrees of freedom (DOF): a pitch rotation $\theta$ and a flap rotation $\gamma$, as shown in Fig. 2 . The pitch axis, $e_{s}$, is parallel to the leading edge and passes by the hinge while the flap axis, $e_{c}$ is parallel to the root of the wing, at a distance $s_{1}$ above it.

The stiffness in both pitch and flap is provided by a thin C75S leaf spring. It is $100 \mathrm{~mm}$ long, $70 \mathrm{~mm}$ large and $0.7 \mathrm{~mm}$ thick. It is clamped linearly to the flat plate and nonlinearly to the roof of the test section of the wind tunnel. Figure 3(a) draws the geometry of the nonlinear root clamps and figure 3(b) plots the nonlinear restoring torque of the pitch DOF. The flap stiffness is linear in the displacement range considered. Finally, a $500 \mathrm{~mm} \times 50 \mathrm{~mm} \times 15 \mathrm{~mm}$ beam is bolted at the junction between the flat plate and the 


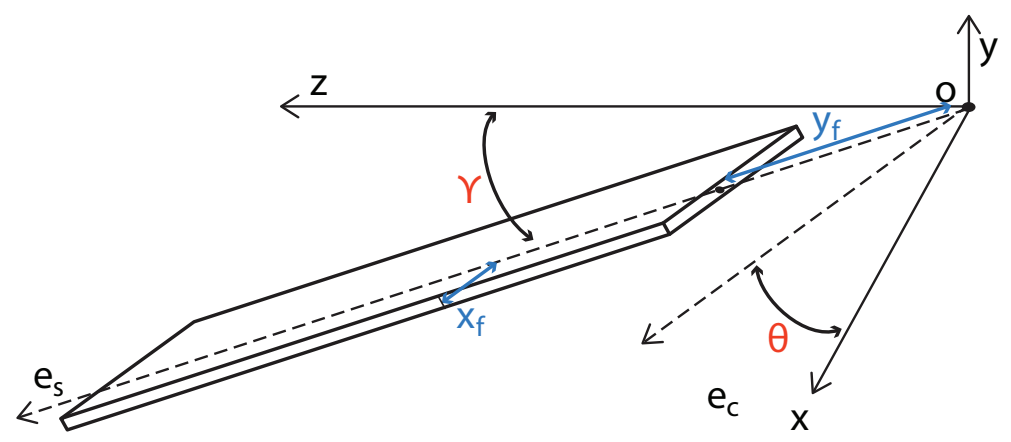

Figure 2: Schematic of the Hancock Wing

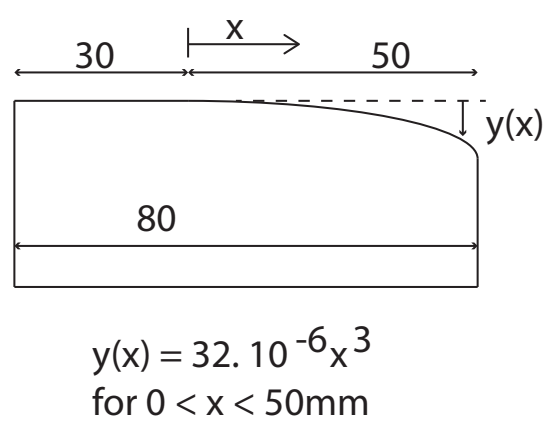

(a) Sketch of the clamps

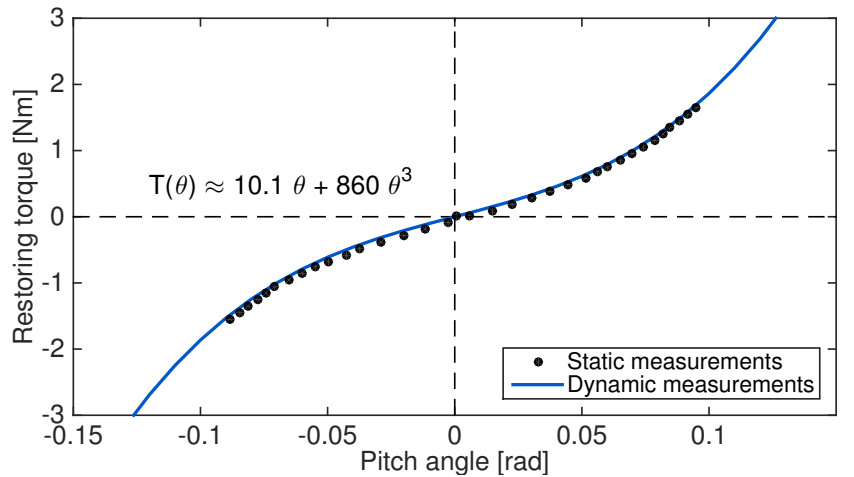

(b) Experimental restoring torque curve of the pitch DOF

Figure 3: Characteristics of the nonlinear clamps

leaf spring (see Fig. 1(a) and 1(b)). It increases the rotational inertia of the system and consequently decreases its flutter speed to the target speed range: [10-15] $\mathrm{m} / \mathrm{s}$. Table 1 summarises all the wind-off characteristics of the system.

The displacements are measured by means of 3 Sick OD2-P300W200I0 laser sensors with a sensitivity of $9.6 \mathrm{mV} / \mathrm{mm}$, a range of $100-500 \mathrm{~mm}$ and a sampling frequency of $1000 \mathrm{~Hz}$. The positions of all the sensors are shown in figure $1(\mathrm{~b})$ and are denoted by $D_{1}, D_{2}$ and $D_{3}$. The measurements from sensors $D_{1}$ and $D_{2}$ are used to calculate the flap angle

$$
\gamma=\arctan \left[\left(D_{1}-D_{2}\right)\left(\Delta s_{\text {las }}\right)^{-1}\right]
$$

while those from sensors $D_{2}$ and $D_{3}$ are used to calculate the pitch angle

$$
\theta=\arctan \left[\left(D_{2}-D_{3}\right)\left(\Delta c_{l a s}\right)^{-1}\right]
$$

Figure 4 displays the system's relevant mode shapes and frequencies identified through a roving hammer test using a single accelerometer placed at the trailing edge of the wingtip and 24 impact locations. The flap mode features a frequency of $0.9 \mathrm{~Hz}$ and a damping of $1 \%$ while the pitch mode features a linear frequency of $3.1 \mathrm{~Hz}$ and a damping ratio of $0.3 \%$. Finally the third mode, which could be considered as a superposition of flapping and plunging motion combined with very little flat plate deformation, was identified to make sure its frequency was high enough to have little impact on the flutter mechanics. This is in fact the case as it features a frequency of $9.6 \mathrm{~Hz}$ and a modal damping ratio of $0.5 \%$. 


\begin{tabular}{|cccc|}
\hline Caracteristic & Symbol & value & Unit \\
\hline \hline Dimensions of the wing: & & & \\
\hline Span & $s$ & 800 & {$[\mathrm{~mm}]$} \\
distance (flap axis - wing root) & $s_{1}$ & 65 & {$[\mathrm{~mm}]$} \\
distance (flap axis - wing tip) & $s_{2}$ & 865 & {$[\mathrm{~mm}]$} \\
Chord & $c$ & 200 & {$[\mathrm{~mm}]$} \\
Half Chord & $b$ & 100 & {$[\mathrm{~mm}]$} \\
Thickness & $t$ & 4 & {$[\mathrm{~mm}]$} \\
Position of the center of mass & $x_{c g}$ & $1 / 2 c$ & {$[\mathrm{~mm}]$} \\
\hline Flap properties & & & \\
\hline Linear stiffness & $K_{\gamma}$ & $\approx 5$ & {$\left[\mathrm{Nm} / \mathrm{rad}^{2}\right]$} \\
Inertia & $I_{\gamma}$ & 0.42 & {$\left[\mathrm{~kg} \mathrm{~m}{ }^{2}\right]$} \\
Damping & $\zeta_{\gamma}$ & $\approx 1$ & {$[\%]$} \\
Frequency & $f_{\gamma}$ & 0.85 & {$[\mathrm{~Hz}]$} \\
\hline Pitch properties & & & \\
\hline Inertia & $I_{\theta}$ & 0.029 & {$\left[\mathrm{~kg} \mathrm{~m}{ }^{2}\right]$} \\
Flexural axis position & $x_{f}$ & $0.3 c$ & {$[\mathrm{~mm}]$} \\
Relative position of $x_{f}$ and $x_{c g}$ & $a$ & $x_{f}-x_{c g}$ & {$[-]$} \\
Linear stiffness coefficient & $K_{\theta}$ & 10.1 & {$[\mathrm{Nm} / \mathrm{rad}$} \\
Quadratic stiffness coefficient & $K_{\theta, 2}$ & $\approx 0$ & {$\left[\mathrm{Nm} / \mathrm{rad}^{2}\right]$} \\
Cubic stiffness coefficient & $K_{\theta, 3}$ & 858 & {$\left[\mathrm{Nm} / \mathrm{rad}^{3}\right]$} \\
Damping & $\zeta_{\theta}$ & $\approx 0.3$ & {$[\%]$} \\
Frequency & $f_{\theta}$ & 3.1 & {$[\mathrm{~Hz}]$} \\
\hline Position of the sensors & & & \\
\hline Distance between $A_{1}$ and $A_{2}$ & $\Delta s_{a c c}$ & 200 & {$[\mathrm{~mm}]$} \\
Distance between $A_{2}$ and $A_{3}$ & $\Delta c_{a c c}$ & 180 & {$[\mathrm{~mm}]$} \\
Distance between $D_{1}$ and $D_{2}$ & $\Delta s_{l a s}$ & 205.5 & {$[\mathrm{~mm}]$} \\
Distance between $D_{2}$ and $D_{3}$ & $\Delta c_{a c c}$ & 168.5 & {$[\mathrm{~mm}]$} \\
\hline
\end{tabular}

Table 1: Wind-off characteristics of the NLPF
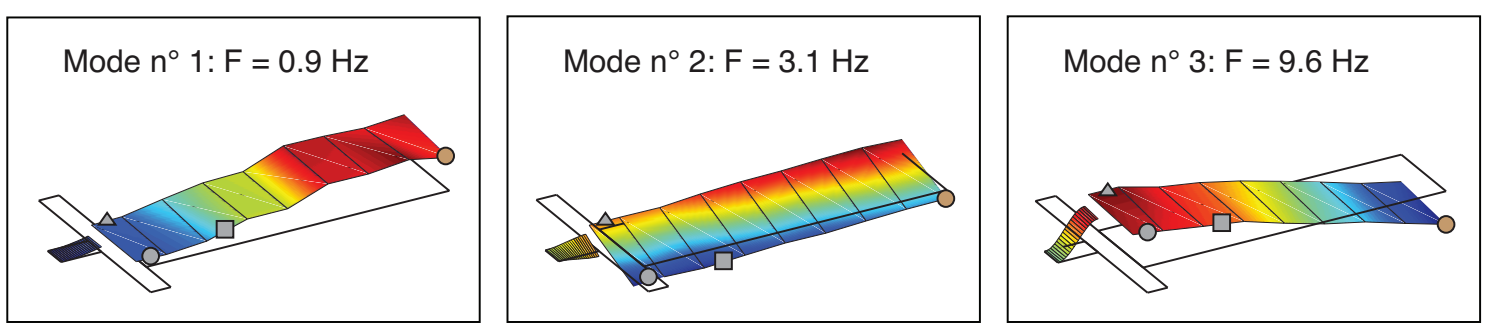

Figure 4: Mode shapes of NLPFW 


\subsection{Reduced order model}

The nonlinear pitch and flap wing is modelled as a two-DOF system, assuming that the flexible modes of the plate do not participate significantly in the observed aeroelastic phenomena. The structural nonlinear equations of motion are

$$
\begin{gathered}
\left(\begin{array}{cc}
I_{\gamma} & S \\
S & I_{\theta}
\end{array}\right)\left(\begin{array}{l}
\ddot{\gamma} \\
\ddot{\theta}
\end{array}\right)+\left(\begin{array}{cc}
c_{\gamma} & c_{\gamma \theta} \\
c_{\gamma \theta} & c_{\theta}
\end{array}\right)\left(\begin{array}{c}
\dot{\gamma} \\
\dot{\theta}
\end{array}\right)+\left(\begin{array}{cc}
k_{\gamma} & k_{\gamma \theta} \\
k_{\gamma \theta} & k_{\theta}
\end{array}\right)\left(\begin{array}{l}
\gamma \\
\theta
\end{array}\right)+\left(\begin{array}{l}
M_{\gamma, N L}(\gamma) \\
M_{\theta, N L}(\theta)
\end{array}\right)=\left(\begin{array}{l}
M_{\gamma, \mathrm{ext}}(t) \\
M_{\theta, \mathrm{ext}}(t)
\end{array}\right) \\
M_{\gamma, N L}=0 \\
M_{\theta, N L}=k_{n l, 3} \theta^{3}
\end{gathered}
$$

where the inertia stiffness and damping parameters are given in table 1 . A nonlinear torque is applied only to the pitch DOF and it is a cubic function of $\theta$.

The flap and pitch aerodynamic moments, $M_{\gamma}$ and $M_{\theta}$ respectively, are computed using strip theory (see for instance Bisplinghoff $\&$ al. $\left.{ }^{[11]}\right)$, which calculates the total 3D aerodynamic loads as sums of 2D loads, $\mathrm{d} L$ and $\mathrm{d} M_{\theta}$, acting on small spanwise strips of width $\mathrm{d} y$. These infinitesimal forces and moments are computed assuming $2 \mathrm{D}$ aerodynamics. The strip theory assumption leads to the following formulation for the flap and pitch aerodynamic moments

$$
\begin{gathered}
M_{\gamma}=-\int_{s_{1}}^{s_{2}} y \mathrm{~d} L(t) \\
M_{\theta}=\int_{s_{1}}^{s_{2}} \mathrm{~d} M_{\theta}(t)
\end{gathered}
$$

$s_{1}$ being the distance between the flap axis and the root of the wing and $s_{2}$ the distance between the flap axis and the tip of the wing. The lift force $\mathrm{d} L$ and pitch moment $\mathrm{d} M_{\theta}$ of a strip can be computed using any 2D unsteady aerodynamic formulation. Unsteady aerodynamic modelling based on Wagner's function ${ }^{[12]}$ was chosen here because the reduced frequency of oscillation $k=\frac{\omega b}{U} \approx 1.5>>0.02$ is too large to use quasi-steady aerodynamics while Theodorsen theory is defined in frequency domain, which makes it difficult to use on a nonlinear system. The growth of circulation around a flat un-cambered airfoil after a step change of incidence is approximated by Wagner's function

$$
\Phi(t)=1-\psi_{1} e^{-\frac{\epsilon_{1} U t}{b}}-\psi_{2} e^{-\frac{\epsilon_{2} U t}{b}}
$$

Integrating equations 5 and 6 with Fung's lift and moment expressions ${ }^{[12]}$ and applying a transformation to replace the wake integrals by aerodynamic state variables ${ }^{[13]}$ yields the complete equations of motion

$$
\begin{aligned}
& {\left[\left(\begin{array}{cc}
I_{\gamma} & S \\
S & I_{\theta}
\end{array}\right)+\rho \pi b^{2}\left(\begin{array}{cc}
\Lambda_{3} / 3 & -a b \Lambda_{2} / 2 \\
-a b \Lambda_{2} / 2 & b^{2} \Lambda_{1}\left(a^{2}+1 / 8\right)
\end{array}\right)\right]\left(\begin{array}{c}
\ddot{\gamma} \\
\ddot{\theta}
\end{array}\right)+} \\
& {\left[\left(\begin{array}{cc}
c_{\gamma} & c_{\gamma \theta} \\
c_{\gamma \theta} & c_{\theta}
\end{array}\right)+\rho \pi U b\left(\begin{array}{cc}
\frac{2}{3} \Phi(0) \Lambda_{3} & b \Lambda_{2}[1 / 2-\Phi(0)(1-a / 2)] \\
-b \Lambda_{2} \Phi(0)(a+1 / 2) & -\Lambda_{1} b^{2}(a-1 / 2)[1+2 \Phi(0)(a+1 / 2)]
\end{array}\right)\right]\left(\begin{array}{c}
\dot{\gamma} \\
\dot{\theta}
\end{array}\right)+} \\
& {\left[\left(\begin{array}{cc}
k_{\gamma} & k_{\gamma \theta} \\
k_{\gamma \theta} & k_{\theta}
\end{array}\right)+\rho \pi U^{2}\left(\begin{array}{cc}
\frac{2}{3} \Lambda_{3}\left[\frac{b \dot{\Phi}(0)}{U}\right] & b \Lambda_{2}\left[\Phi(0)+\frac{b \dot{\Phi}(0)}{U}(1 / 2-a)\right] \\
-b \Lambda_{2}\left[\frac{b \dot{\Phi}(0)}{U}(a+1 / 2)\right] & -2 \Lambda_{3}(a+1 / 2)\left[\Phi(0)-\frac{b \dot{\Phi}(0)}{U}(a-1 / 2)\right]
\end{array}\right)\right]\left(\begin{array}{l}
\gamma \\
\theta
\end{array}\right)+} \\
& {\left[\pi \rho U^{3}\left(\begin{array}{cc}
-2 \epsilon_{1}^{2} \psi_{1} \Lambda_{3} / 3 b & \epsilon_{1}^{2} \psi_{1} \Lambda_{2}(2 a+1) / 2 \\
-2 \epsilon_{2}^{2} \psi_{2} \Lambda_{3} / 3 b & \epsilon_{2}^{2} \psi_{2} \Lambda_{2}(2 a+1) / 2 \\
\Lambda_{2}\left(\epsilon_{1} \psi_{1}-\epsilon_{1}^{2} \psi_{1} / 2+a \epsilon_{1}^{2} \psi_{1}\right) & -2 b \epsilon_{1} \psi_{1} \Lambda_{1}(a+1 / 2)\left(\epsilon_{1}(a-1 / 2)+1\right) \\
\Lambda_{2}\left(\epsilon_{2} \psi_{2}-\epsilon_{2}^{2} \psi_{2} / 2+a \epsilon_{2}^{2} \psi_{2}\right) & -2 b \epsilon_{2} \psi_{2} \Lambda_{1}(a+1 / 2)\left(\epsilon_{2}(a-1 / 2)+1\right)
\end{array}\right)^{T}\right]\left(\begin{array}{l}
w_{1} \\
w_{2} \\
w_{3} \\
w_{4}
\end{array}\right)=} \\
& {\left[\left(\begin{array}{c}
M_{\gamma, \text { ext }}(t) \\
M_{\theta, \text { ext }}(t)
\end{array}\right)+2 \pi \rho U b \dot{\Phi}(t)\left(\begin{array}{c}
\Lambda_{3} \gamma(0) / 3+\left(\frac{3 b}{2}-x_{f}\right) \Lambda_{2} \theta(0) / 2 \\
-(a+1 / 2) b\left[\Lambda_{2} \gamma(0) / 2+\left(\frac{3 b}{2}-x_{f}\right) \Lambda_{1} \theta(0)\right]
\end{array}\right)-\left(\begin{array}{c}
0 \\
k_{n l, 3} \theta^{3}
\end{array}\right)\right]}
\end{aligned}
$$

with $\Lambda_{j}=s_{2}^{j}-s_{1}^{j}$. The left hand side of Eq. 8 includes inertia, damping, stiffness terms and the aerodynamic state proportionality matrix. The right hand side comprises three terms, the external loads (set to zero since self-excited oscillations are under investigation), a transient term, which is also set to zero since it quickly decays in time, and a term related to the structural nonlinearities. The nonlinear equations of motion are solved using a numerical continuation algorithm based on a finite difference formulation ${ }^{[14]}$. 


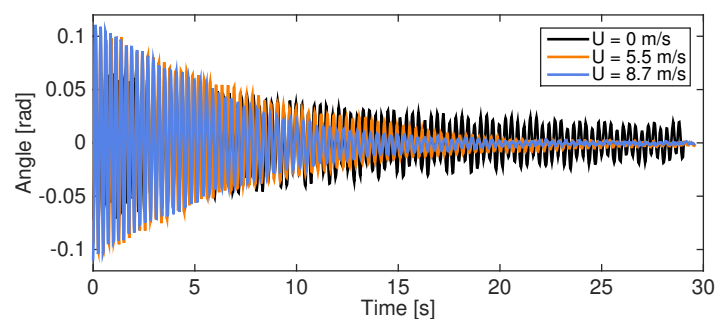

(a) Sub critical pitch response

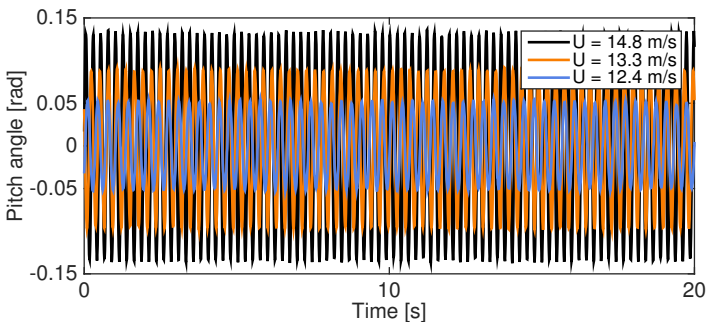

(b) Super critical pitch response

Figure 5: Time series of the pitch response of the system at sub-critical and super-critical airspeeds

\section{AEROELASTIC ANALYSIS}

The system's aeroelastic behaviour is first studied at sub-critical airspeeds, where the average damping is positive and leads to decaying motions, as demonstrated by the pitch response time histories plotted in figure 5 (a) for airspeeds of $0,5.5$ and $8.7 \mathrm{~m} / \mathrm{s}$. At higher airspeeds, the response is a self-excited limit cycle oscillation (LCO). This behaviour is demonstrated in figure 5(b) which plots pitch response time histories at airspeeds of 12.413 .3 and $14.8 \mathrm{~m} / \mathrm{s}$.

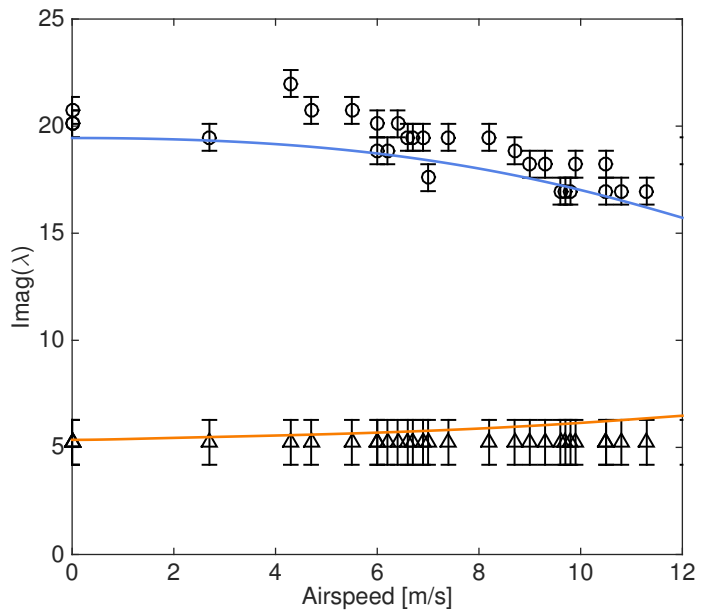

(a) Pulsation

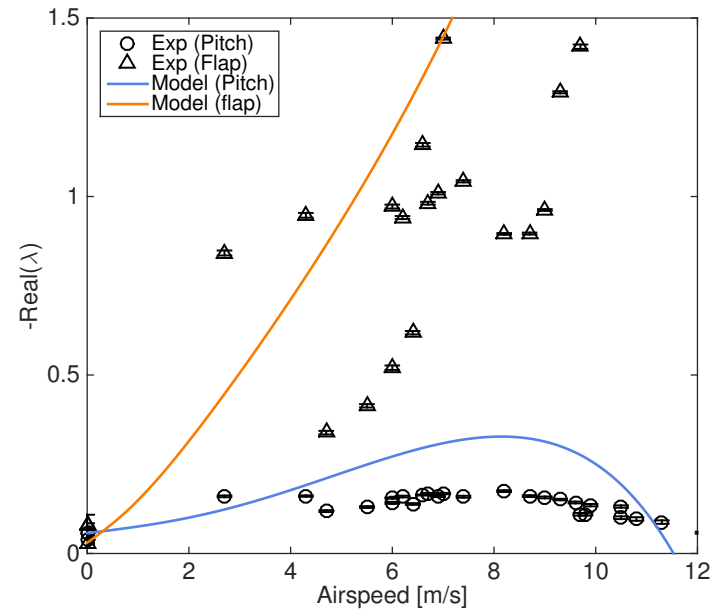

(b) Decay rate

Figure 6: Variation of the pitch and flap modal parameters with the airspeed

Figure 6 displays the variation of the modal parameters of the pitch and flap modes of the NLPFW with airspeed according to experimental measurements (black dots) and the analytical model (plain lines). The imaginary part of the response -identified using Fast Fourier Transform- is accurately reproduced by the model and shows the typical features of an aeroelastic system undergoing flutter: the frequency gap between the two modes decreases as the airspeed is increased until the modes are close enough to interact and cause flutter. The real part -identified using an exponential fitting of the Hilbert transform of the response- is also typical of aeroelastic systems: the flap damping strongly increases while the pitch damping increases at first then decreases until it drops to zero and flutter occurs. In this case, the model seems to over-estimate the damping, however it must be noted that damping is very difficult to identify and even harder to model; considering the simplicity of the model, the estimate is satisfactory.

Once the flutter speed is reached, the average damping of the system drops to zero and limit cycle oscillations are observed. Figure 


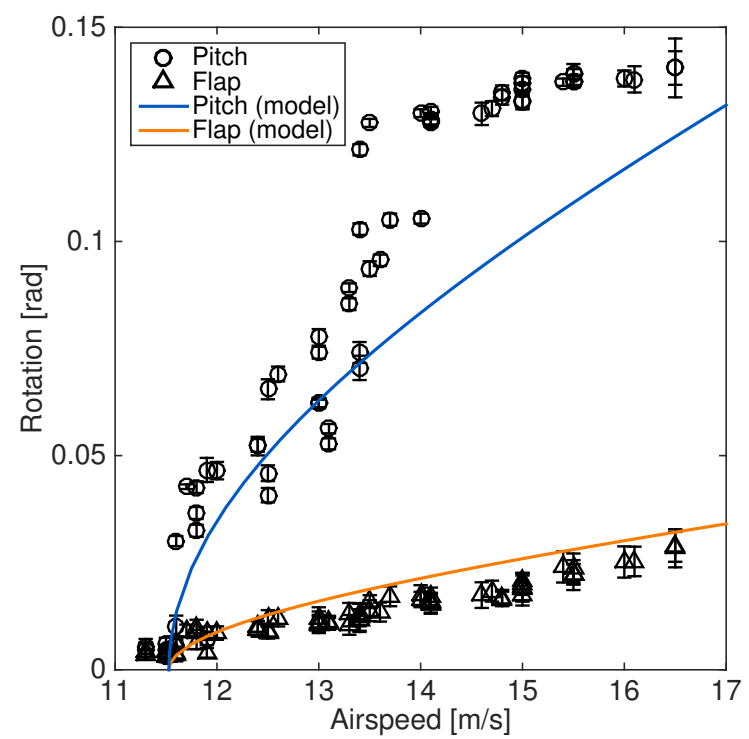

(a) Pitch and Flap LCO amplitude

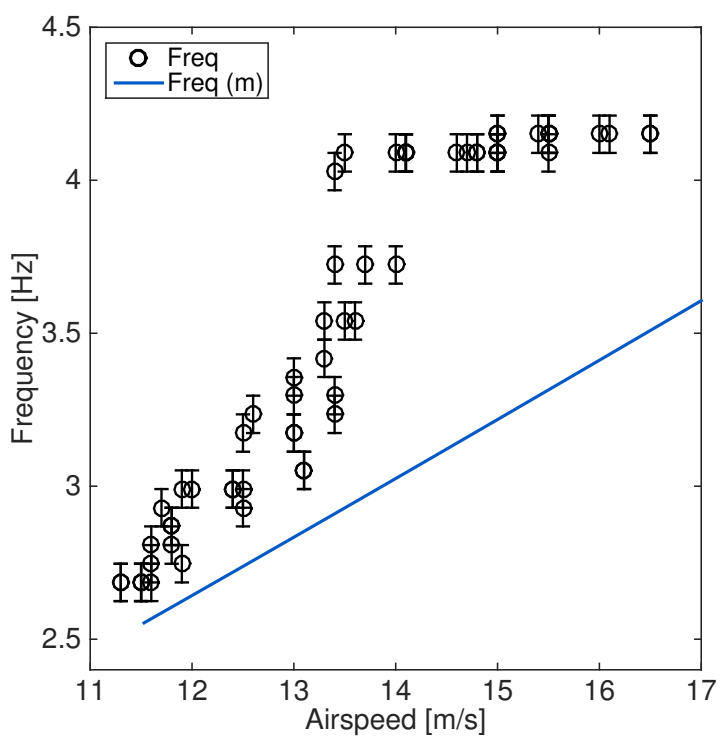

(b) LCO frequency

Figure 7: Experimental and numerical bifurcation diagrams of the primary system

7 (a) displays the bifurcation diagrams in pitch and flap angle of the system obtained from wind tunnel observations (triangles and dots) and from the numerical continuation analysis of equation 8. The airspeed was increased then decreased in the wind-tunnel to look for hysteresis effects but none were found. Both experimental and numerical results exhibit a supercritical Hopf bifurcation at $11.5 \mathrm{~m} / \mathrm{s}$, followed by increases in LCO amplitude and frequency, consistent with cubic hardening stiffness. At $13.5 \mathrm{~m} / \mathrm{s}$ a discontinuity occurs in the experimental pitch measurements; both the amplitude and frequency increase sharply. This phenomenon is due to dynamic stall and is overlooked by the reduced order model, which uses linear aerodynamics. The flap displacement undergoes a gentle increase in amplitude that is predicted with reasonably accuracy by the model. Because of the coupling between the pitch and the flap modes, at $13.5 \mathrm{~m} / \mathrm{s}$ a jump is observed also in the flap response. However, the increase in amplitude is not significant.

The variation of the LCO frequency with airspeed is shown in figure 7 (b). Flutter starts at $2.6 \mathrm{~Hz}$ in the wind tunnel, then the LCO frequency increases almost linearly with the airspeed until $13.5 \mathrm{~m} / \mathrm{s}$, where it jumps to $4.2 \mathrm{~Hz}$. The model predicts a linear increase of the frequency with an absolute error around $0.3 \mathrm{~Hz}$, until the jump where it fails because it cannot represent nonlinear aerodynamic effects.

Summarising, the NLFPW undergoes classical flutter at $11.5 \mathrm{~m} / \mathrm{s}$, followed by a gently increase in pitch amplitude until $13.5 \mathrm{~m} / \mathrm{s}$, where a jump is observed. These aeroelastic phenomena are predicted with reasonable accuracy by the model from $0 \mathrm{~m} / \mathrm{s}$ to 13.5 $\mathrm{m} / \mathrm{s}$. This speed range is used to assess the effect of a LTVA on the system.

\section{LTVA DESIGN}

Figure 8(a) displays the experimental absorber configuration and figure $8(\mathrm{~b})$ the numerical model. The LTVA consists of a flexible beam of length $r_{l t v a}=0.1 \mathrm{~m}$, clamped on one end at a distance $r_{\theta}=0.125 \mathrm{~m}$ from the pitch axis and $r_{\gamma}=0.05 \mathrm{~m}$ from the flap axis, and a mass $m_{l t v a}$ attached at the free end of the beam. The beam provides both stiffness, $k_{l t v a}$, and damping, $c_{l t v a}$.

The LTVA is modelled mathematically as a mass-spring-damper system with a rotational DOF, $\xi$, in the plane $\phi$ around the LTVA axis, which is parallel to the pitch axis. Assuming small displacements, the mathematical and experimental configurations are similar. 


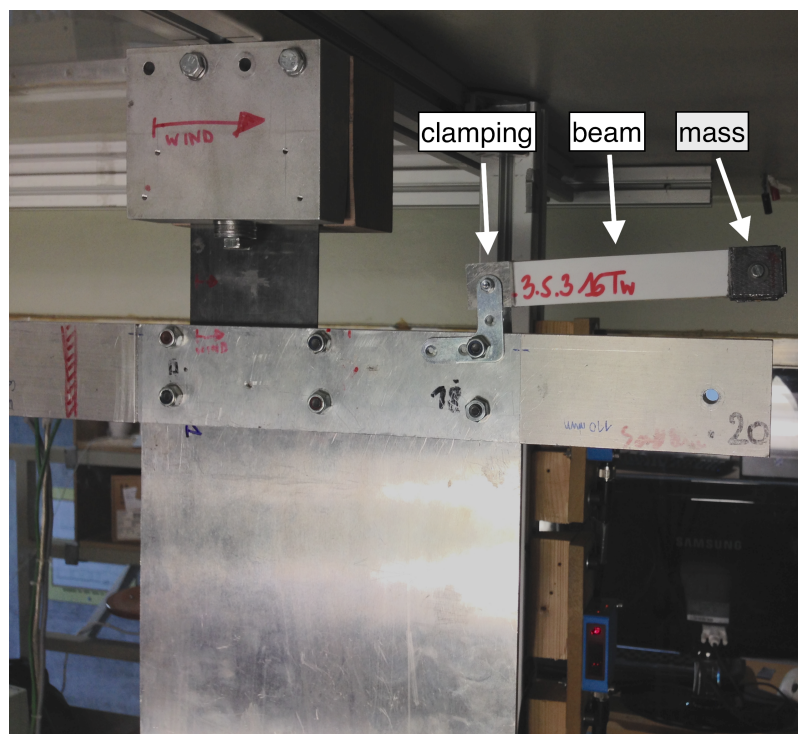

(a) Picture of the experimental LTVA attached to the primary (b) system

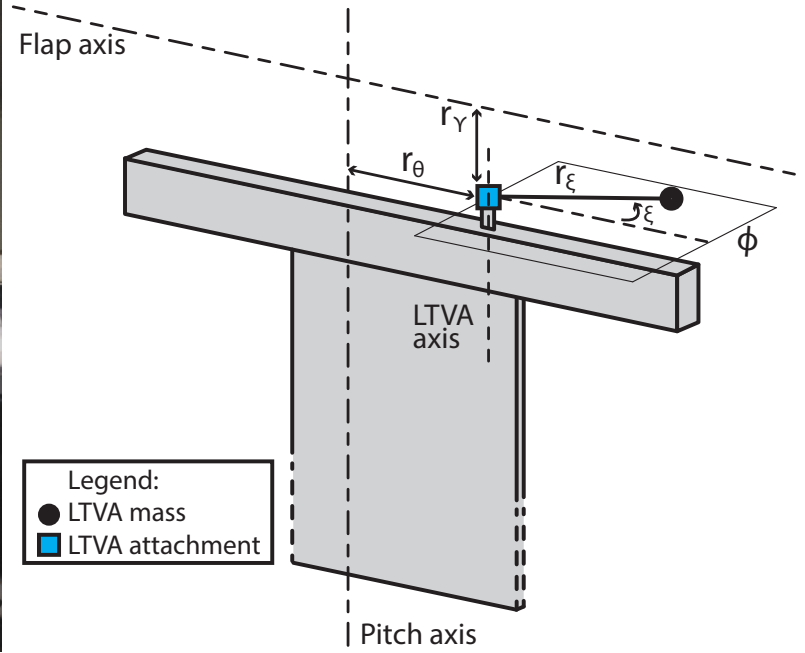

(b) Sketch of the model of the LTVA attached to the primary system

Figure 8: Experimental and modelled LTVA configurations

The natural frequency and damping ratio of the LTVA are given by

$$
\begin{aligned}
f_{l t v a} & =\frac{1}{2 \pi} \sqrt{k_{\text {ltva }} / I_{\text {ltva }}}=\frac{1}{2 \pi} \sqrt{k_{\text {ltva }} /\left(m_{\text {ltva }} r_{\text {ltva }}^{2}\right)} \\
\epsilon_{\text {ltva }} & =c_{\text {ltva }} /\left(2 \sqrt{k_{\text {ltva }} I_{\text {ltva }}}\right)
\end{aligned}
$$

\subsection{LTVA equations of motion}

Adopting the Euler-Lagrange equation, the equations of motion of the coupled 3-DOFs are derived. The resulting inertia, damping and stiffness matrices are given by

$$
\begin{aligned}
& M_{s}=\left(\begin{array}{ccc}
I_{\gamma} & S & 0 \\
S & I_{\theta} & 0 \\
0 & 0 & 0
\end{array}\right)+m_{l t v a}\left(\begin{array}{ccc}
r_{\gamma}^{2} & r_{\gamma}\left(r_{\theta}+r_{l t v a}\right) & r_{\gamma} r_{l t v a} \\
r_{\gamma}\left(r_{\theta}+r_{l t v a}\right) & \left(r_{\theta}+r_{l t v a}\right)^{2} & \left(r_{\theta}+r_{l t v a}\right) r_{l t v a} \\
r_{\gamma} r_{l t v a} & \left(r_{\theta}+r_{l t v a}\right) r_{l t v a} & r_{l t v a}^{2}
\end{array}\right) \\
& C_{s}=\left(\begin{array}{ccc}
c_{\gamma} & 0 & 0 \\
0 & c_{\theta} & 0 \\
0 & 0 & 0
\end{array}\right)+c_{l t v a}\left(\begin{array}{ccc}
0 & 0 & 0 \\
0 & 0 & 0 \\
0 & 0 & 1
\end{array}\right) \\
& K_{s}=\left(\begin{array}{ccc}
k_{\gamma} & 0 & 0 \\
0 & k_{\theta} & 0 \\
0 & 0 & 0
\end{array}\right)+k_{l t v a}\left(\begin{array}{ccc}
0 & 0 & 0 \\
0 & 0 & 0 \\
0 & 0 & 1
\end{array}\right)
\end{aligned}
$$

where the contributions from the NLPFW and LTVA have been separated. The aerodynamic effects are assumed unchanged after the addition of the LTVA, i.e. the aerodynamic effects on the LTVA are neglected. Consequently, the full aeroelastic equations of motion, including the absorber, are still given by Eq. 8, after substituting the structural inertia, damping and stiffness matrices from those in Eq. 10. 


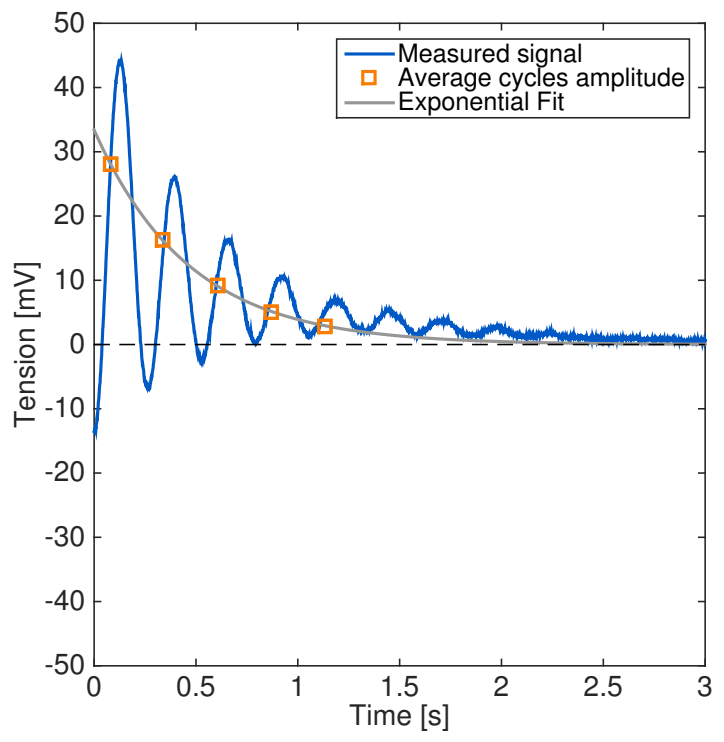

(a) Free decay response of the LTVA 30-50-30-16T

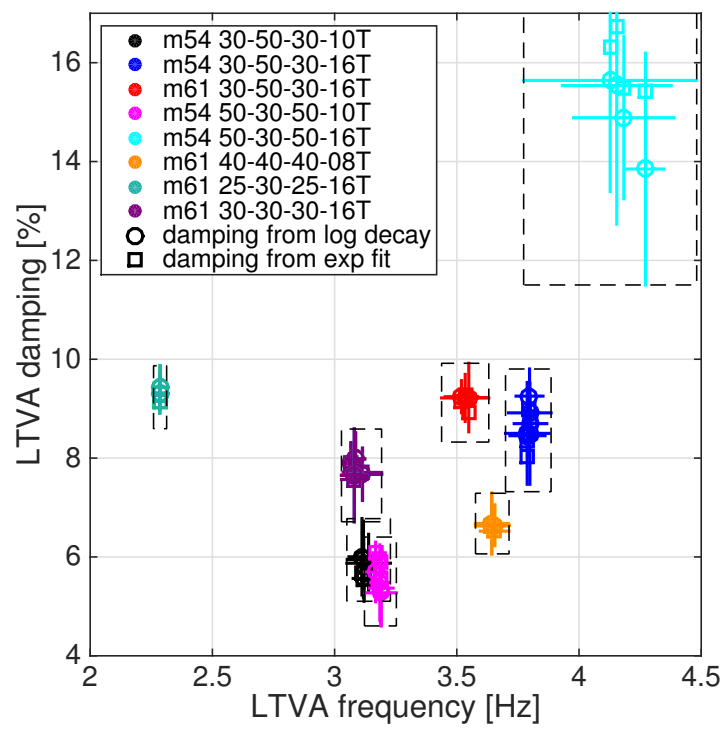

(b) Identification results of all the experimental LTVAs

Figure 9: Experimental identification of the LTVAs

\subsection{Experimental LTVA design and identification}

A number of different LTVAs were built. In order to achieve high damping ratios, the LTVA beams were made of a sandwich of three polymer sheets, with thicknesses varying from 0.3 to $0.5 \mathrm{~mm}$, and 10 or 16 layers of viscoelastic tape wrapped around the central polymer sheet. The thickness of the polymer sheets and the number of layers of viscoelastic tape affected both the stiffness and the damping of the absorber.

The LTVAs were characterised by performing free response vibration tests such as the one displayed in figure 9(a) for the LTVA $\mathrm{m} 54-30-50-30-16 \mathrm{~T}$. The amount of damping in the system and the shearing between the layers lead to asymmetric oscillations and drift so a special peak-picking routine was developed to measure the damping and the frequency. Instead of tracking the changes of sign to identify the successive periods, each cycle's period is computed from the extrema of the signal and the logarithmic decrement is computed from the average amplitude of each cycle defined as $\left(A_{\max }-A_{\min }\right) / 2$. An exponential fitting of the amplitude at successive cycles was also carried out to identify the damping and the two methods gave similar damping estimates. To ensure that the drift of the response had little effect on the damping identification procedure, all the free response tests were repeated with both positive and negative initial displacements.

Figure 9(b) plots the frequencies and dampings obtained from of all the LTVA free response tests. The frequency varies from $2.3 \mathrm{~Hz}$ to $4.5 \mathrm{~Hz}$ and the damping between $5 \%$ and $16 \%$. The names mxx-yy-yy-yy-zzT are related to the composition of the beam: $\mathrm{mxx}$ gives the mass in grams, yy denotes the thicknesses of the polymer sheets and $z z T$ indicates the amount of viscoelastic tape layers placed in the sandwich. The errorbars indicate the standard deviation of the damping and frequencies measured from the free response tests. The LTVA with the higher frequency also featured the higher damping. It was found impossible to build an LTVA with low frequency and high damping, as increasing the number of viscoelastic tape layers increased both the damping and stiffness of the beams. Increasing the mass was not always possible because a high enough mass would cause buckling. 


\section{NUMERICAL AND EXPERIMENTAL AEROELASTIC ANALYSIS OF THE PRIMARY SYSTEM COUPLED WITH THE LTVA}

\subsection{Effect of the LTVA on the flutter speed}

In the wind tunnel the critical flutter speed was defined as the first airspeed at which LCOs were clearly observed. Both increasing and decreasing airspeed tests were carried out to search for hysteresis effects. No such effects were observed, suggesting that all bifurcations were supercritical.

The flutter speed was also estimated from the mathematical model, for a very wide range of LTVA frequencies and damping ratios. The solid surface on figure 10 plots the results of this calculation; the flutter speed is plotted as a ratio of flutter speeds with and without LTVA. All the results in the figure were obtained for a LTVA mass of 64 grams $(\approx 2.3 \%$ mass ratio). The experimental flutter speeds are plotted on the same axes. Figure 10(b) plots the same data seen from above, so that the effect of both frequency and damping ratio on the flutter speed is displayed.

The mathematical model predicts a maximum increase in flutter speed of $34 \%$. This optimum is obtained at $1.892 \mathrm{~Hz}$ and 14.47 $\%$ damping and quickly decreases as the LTVA gets detuned. The flutter speed is very sensitive to LTVA frequency; a frequency change of $0.1 \mathrm{~Hz}$ can lead to a decrease in non-dimensional flutter speed from 1.34 to 1.24 . The sensitivity to damping ratio is much lower. The LTVAs tested in the wind tunnel showed similar optimum flutter speeds, however the results appeared to be far less sensitive to detuning than in the case of the mathematical model. The cyan LTVA, for instance, was supposed to be completely detuned according to the model yet it yielded a commendable increase in flutter speed of $17 \%$ in the wind-tunnel. There are several possible reasons for the difference between the detuning behaviour of the experiment and the mathematical model:

1. The LTVA structural damping may be nonlinear in practice but is modelled as linear.

2. The flow around the LTVA is not modelled but may have an affect on the absorber's effectiveness.

3. The difference between the modelled LTVA (rigid body rotation) and the experimental absorber (cantilever beam) may be too pronounced.

Finally it must be noted that the higher the damping the lower the sensitivity to frequency tuning. For a real life application it might be better to tune the LTVA to a damping higher than its optimum value.

\subsection{Effect of the LTVA on the subcritical response of the system}

The mechanism leading to an optimum tuning was investigated by studying numerically the effect of the LTVA on the variations of the modal frequencies and damping ratios with airspeed. These are variations are plotted in figure 11 . The absorbers considered have mass $m_{l t v a}=64$ grams, damping $\zeta_{l t v a}=14.47 \%$ and frequencies $1.892 \mathrm{~Hz}, 1.8 \mathrm{~Hz}$ and $2.0 \mathrm{~Hz}$ respectively from top to bottom.

Figures 11(a) and 11(b) display the results with an optimum LTVA. The system with absorber has three modes:

- Mode a: mostly flap motion (red).

- Mode b: in phase pitch and LTVA motion (blue).

- Mode c: out of phase pitch and LTVA motion (orange).

The absorber splits the uncontrolled system's pitch mode (black squares) into modes b and c (orange and blue) and has very low effect on the flap mode (black circles and red), because the flap inertia is larger than the pitch inertia and the LTVA is close to the flap axis and far from the pitch axis. Flutter occurs at $15.58 \mathrm{~m} / \mathrm{s}$ on the system with LTVA. At flutter modes $\mathrm{b}$ and $\mathrm{c}$ have the same frequency and are essentially a single mode. The damping figure shows that again, modes $b$ and $c$ nearly merge at flutter. 
Figures $11(\mathrm{c})$ \& $11(\mathrm{~d})$ plot the effect of a slightly undertuned LTVA $(1.8 \mathrm{~Hz})$. In this case mode $\mathrm{c}$ leads to flutter alone and does not merge with mode $b$ at flutter. Figures $11(\mathrm{e})$ \& $11(\mathrm{f})$ finally show the effect of a slightly overtuned LTVA $(2.0 \mathrm{~Hz})$. In this case, mode $b$ leads to flutter alone and once again no frequency matching of modes $b$ and $c$ occurs.

The fact that, for optimal LTVA tuning, modes b and c undergo flutter simultaneously, resembles the classical Den Hartog's criterion for optimisation of LTVA for forced vibration, where the optimum corresponds to two equally high resonant peaks. This suggests that equating the flutter speed of the pitch modes could indeed be used to calculate the optimal tuning. Although up to now this conclusion is supported only by numerical calculations, in ${ }^{[15]}$ a similar result was obtained for a LTVA applied to a van der Pol-Duffing oscillator.

\subsection{Effect of the LTVA on the supercritical response of the system}

Figure 12 displays experimental and numerical bifurcation diagrams in pitch LCO amplitude (12(a)), flap LCO amplitude (12(b)) and LCO frequency $(12(\mathrm{c}))$. The circles correspond to wind tunnel experiments with velocity sweeps up and down that did not show any hysteretic behaviour in any of the cases investigated. The thick lines show continuation computations from the model with three different LTVA frequencies, namely $1.8,1.89$ and $2.0 \mathrm{~Hz}$, where $1.89 \mathrm{~Hz}$ corresponds to the optimal tuning.

The pitch bifurcation diagram plotted in figure 12(a) already provides most of the information. The model showed that the $1.8 \mathrm{~Hz}$ LTVA (dark blue) improves the flutter speed and barely changes the shape of the bifurcation. In this case the absorber is already detuned when flutter occurs. The $1.89 \mathrm{~Hz}$ LTVA (dark red) is the optimum case for increasing the flutter speed, however it features a narrow region of bi-stable solutions due to fold bifurcations occurring when the absorber is detuned. Finally the $2.0 \mathrm{~Hz}$ absorber (orange) leads to flutter at $14.3 \mathrm{~m} / \mathrm{s}$ then decreases the amplitude of the ensuing LCOs until it gets detuned and the response jumps to a large amplitude limit cycle.

The experiments showed similar performance in flutter speed however no detuning was observed in the wind tunnel and the shape of the bifurcation branches is quite different because of the nonlinear damping, the effect of the airflow on the absorber and the difference in LTVA geometry. The light blue and orange curves correspond to the same LTVA beam with masses of 64 and 70 grams respectively. They show that an increase in mass of just $10 \%$ leads to a decrease in LCO amplitude of roughly $25 \%$. Moreover the heavier case leads to a smoother amplitude variation with airspeed close to the flutter speed. The purple line corresponds to the LTVA with the lowest frequency we could achieve, which should be the best one according to the model. In practice however it showed performance similar to the blue one. Furthermore, the maximum airspeed tested with this LTVA was lower than usual due to impacts phenomena between the absorber and the primary structure. Finally, the green curve corresponds to another intermediate LTVA that should have performed better than the blue one, according to the model, but which showed slightly worse performance.

Figure 12(b) displays the bifurcation diagrams of the system in flap amplitude. The model shows that the undertuned (dark blue) and optimum LTVAs (dark red) rapidly reach the amplitude of the system without absorber, and have a detrimental effect on the flap response. The overtuned LTVA (orange) leads to oscillations of larger amplitude until it is detuned, then it jumps down to the amplitude experienced by the system the with other absorbers. The experiments, on the other hand, featured a smooth increase in amplitude and a better performance than the system without absorber up to $16.5 \mathrm{~m} / \mathrm{s}$. Beyond this airspeed we did not take any measurements without absorber for safety reasons.

The variation of the LCO frequency plotted in figure 12(c) features significant differences after the addition of the LTVA. According to the experimental results, all the absorbers decreased the frequency of the oscillations. Furthermore, the limit cycle frequency varied very little with airspeed. The mathematical model showed that only the optimum and overtuned LTVAs decrease the frequency, for as long as they remain tuned (i.e. near the flutter speed and at low LCO amplitudes). The undertuned absorber has very little effect on the frequency. Once again, it appears that the experimental LTVAs remain tuned over a much wider airspeed range than the mathematical ones.

Considering the uncertainties in the LTVA identification and the complexity of the phenomena that occur in the wind tunnel, the predictions of the model are satisfactory. Finally, none of the experimental bifurcation diagrams performed with a LTVA attached to the system exhibited the jump due to stall-flutter. The increased damping and lower oscillation frequency is probably the reason. 


\section{CONCLUSIONS}

The main purpose of this study was to demonstrate experimentally the effectiveness of the LTVA in delaying flutter and the appearance of limit cycle oscillations. Both the experiments and the mathematical model showed an improvement of about $30 \%$ of the flutter speed with a damper mass of around $2.3 \%$ of the mass of the full system.

The mathematical model demonstrated that optimal flutter delay occurs when the LTVA is tuned such that two of the system modes flutter simultaneously at the same airspeed. However, the effectiveness of the LTVA decreases steeply as the natural frequency of the absorber moves away from an optimum tuned value.

The major difference between the model and the experiments was the sensitivity of the LTVA to its tuning parameters. Absorbers that were totally detuned according to the model showed very good performance in the wind tunnel, which is encouraging for future experimental studies and applications. Furthermore, all the LTVAs suppressed the stall flutter phenomenon that occurs in the uncontrolled system.

Finally, it must be noted that this study considered a single LTVA position. The effects of the position of the LTVA on optimum tuning rules and effectiveness will be investigated in the future. Furthermore, the effect of the introduction of a nonlinear restoring force in the LTVA (leading to a nonlinear tuned vibration absorber ${ }^{[15-17]}$ ) will be assessed, with the objective of improving the supercritical response of the system.

\section{ACKNOWLEDGMENTS}

The authors would like to acknowledge the financial support of the European Union (ERC Starting Grant NoVib 307265).

\section{References}

[1] Nagase, T. and Hisatoku, T. (1992). Tuned-pendulum mass damper installed in crystal tower. The Structural Design of Tall Buildings, 1(1), 35-56.

[2] Lu, X., Li, P., Guo, X., et al. (2014). Vibration control using atmd and site measurements on the shanghai world financial center tower. The Structural Design of Tall and Special Buildings, 23(2), 105-123.

[3] Gu, M., Chang, C., Wu, W., et al. (1998). Increase of critical flutter wind speed of long-span bridges using tuned mass dampers. Journal of Wind Engineering and Industrial Aerodynamics, 73(2), 111-123.

[4] Dallard, P., Fitzpatrick, A., Flint, A., et al. (2001). The london millennium footbridge. Structural Engineer, 79(22), 17-21.

[5] Gu, M., Chang, C., Wu, W., et al. (1998). Increase of critical flutter wind speed of long-span bridges using tuned mass dampers. Journal of Wind Engineering and Industrial Aerodynamics, 73(2), 111-123.

[6] Lin, Y.-Y., Cheng, C.-M., and Lee, C.-H. (2000). A tuned mass damper for suppressing the coupled flexural and torsional buffeting response of long-span bridges. Engineering structures, 22(9), 1195-1204.

[7] Chen, X. and Kareem, A. (2003). Efficacy of tuned mass dampers for bridge flutter control. Journal of Structural Engineering, 129(10), 1291-1300.

[8] Casalotti, A., Arena, A., and Lacarbonara, W. (2014). Mitigation of post-flutter oscillations in suspension bridges by hysteretic tuned mass dampers. Engineering Structures, 69, 62-71.

[9] INGRAM, C. and SZWARC, W. (1976). Passive flutter suppression. Journal of Aircraft, 13(7), 542-543.

[10] Karpel, M. (1981). Design for active and passive flutter suppression and gust alleviation, vol. 3482. National Aeronautics and Space Administration, Scientific and Technical Information Branch.

[11] Bisplinghoff, R., Ashley, H., and Halfman, R. (1996). Aeroelasticity. Dover Publications Inc.

[12] Fung, Y. (1993). An introduction to the Theory of Aeroelasticity. Dover Publications Inc. 
[13] Lee, B., Gong, L., and Wong, Y. (1997). Analysis and computation of nonlinear dynamic response of a two-degree-of-freedom system and its application in aeroelasticity. Journal of Fluids and Structures, 11, 225-246.

[14] Dimitriadis, G. (2008). Numerical continuation of aeroelastic systems: Shooting vs finite difference approach. In RTO-MPAVT-152 Limit Cycle Oscillations and Other Amplitude-Limited Self-Excited Vibrations, AVT-152-025. Loen, Norway.

[15] Habib, G. and Kerschen, G. (2015). Suppression of limit cycle oscillations using the nonlinear tuned vibration absorber. In Proceedings of the Royal Society of London A: Mathematical, Physical and Engineering Sciences, vol. 471. The Royal Society, p. 20140976.

[16] Habib, G., Detroux, T., Viguié, R., et al. (2015). Nonlinear generalization of den hartog»ș s equal-peak method. Mechanical Systems and Signal Processing, 52, 17-28.

[17] Detroux, T., Habib, G., Masset, L., et al. (2015). Performance, robustness and sensitivity analysis of the nonlinear tuned vibration absorber. Mechanical Systems and Signal Processing, 60, 799-809. 


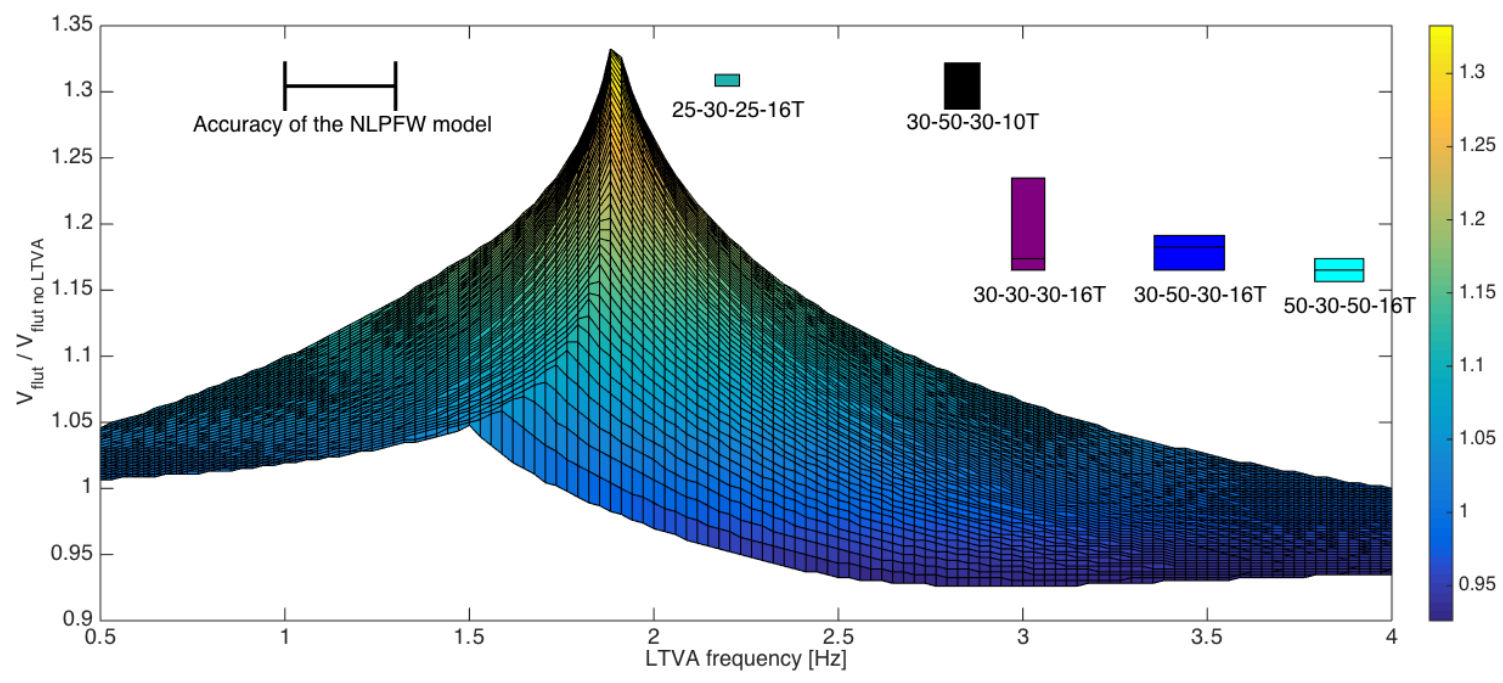

(a) 3D plot of the flutter speed of the NLPFW as a function of the LTVA tuning

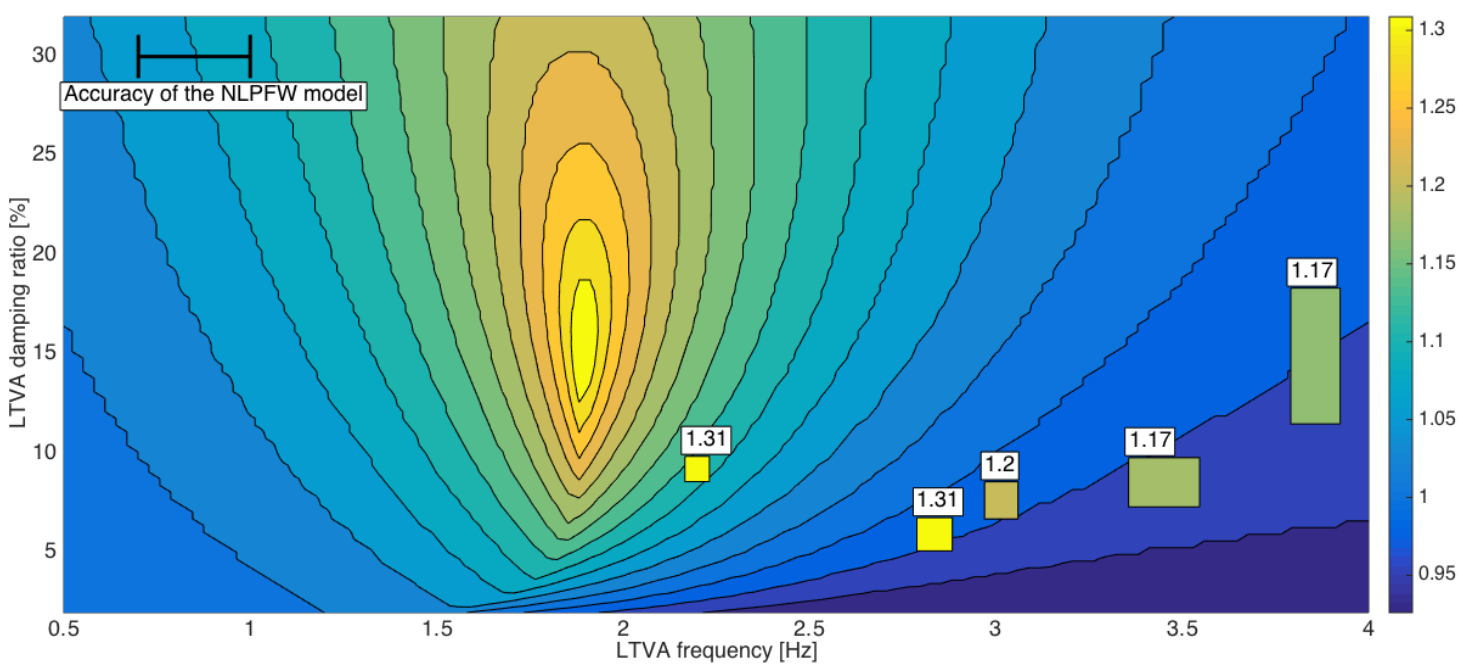

(b) 2D plot of the flutter speed of the NLPFW as a function of the LTVA tuning

Figure 10: Effect of the numerical and experimental LTVA on the flutter speed 


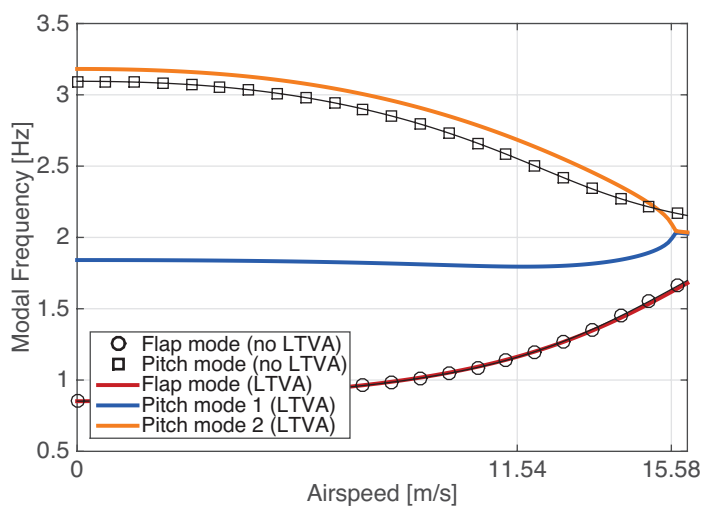

(a) Modal frequency with $f_{l t v a}=1.89 H z, \zeta_{l t v a}=14.47 \%$

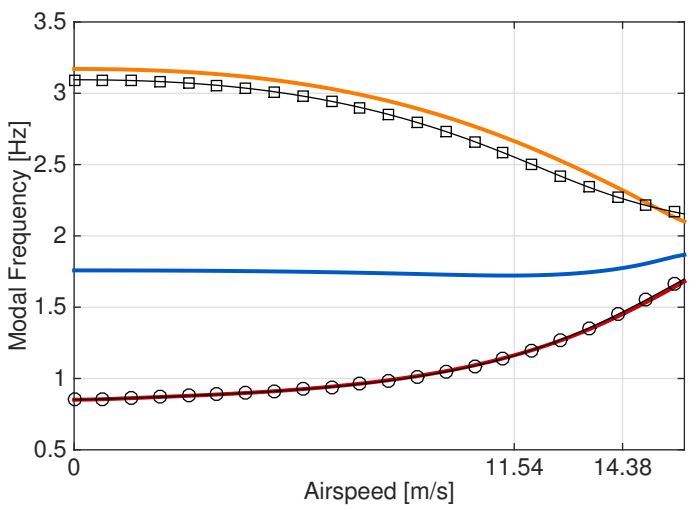

(c) Modal frequency with $f_{l t v a}=1.8 \mathrm{~Hz}, \zeta_{l t v a}=14.47 \%$

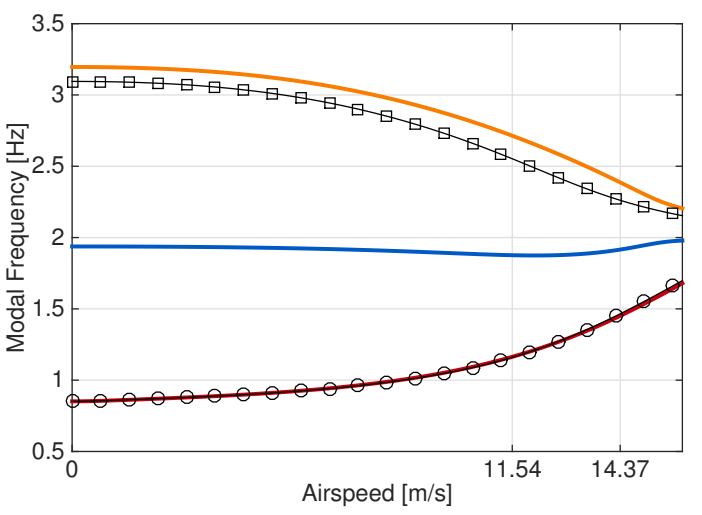

(e) Modal frequency with $f_{\text {ltva }}=2.0 \mathrm{~Hz}, \zeta_{\text {ltva }}=14.47 \%$

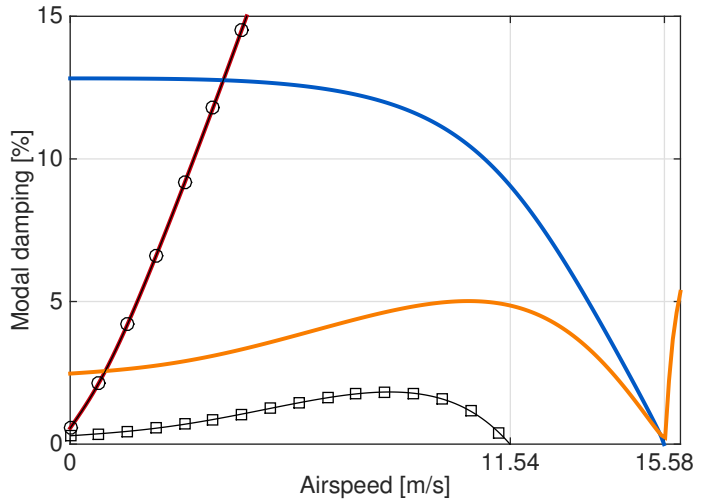

(b) Modal damping with $f_{\text {ltva }}=1.89 \mathrm{~Hz}, \zeta_{\text {ltva }}=14.47 \%$

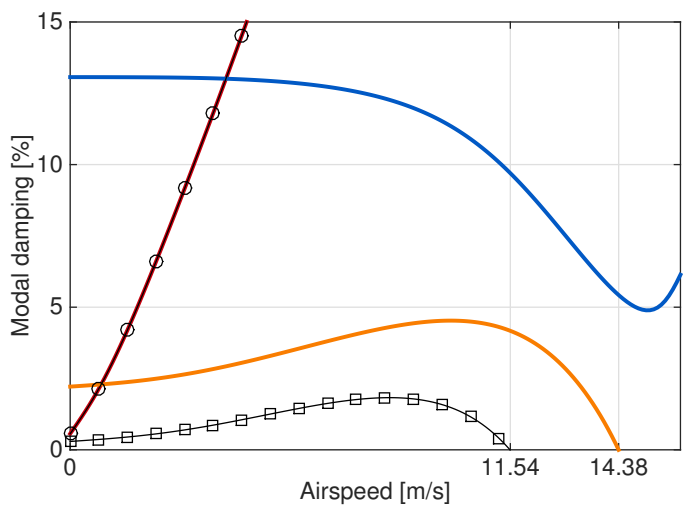

(d) Modal damping with $f_{l t v a}=1.8 \mathrm{~Hz}, \zeta_{l t v a}=14.47 \%$

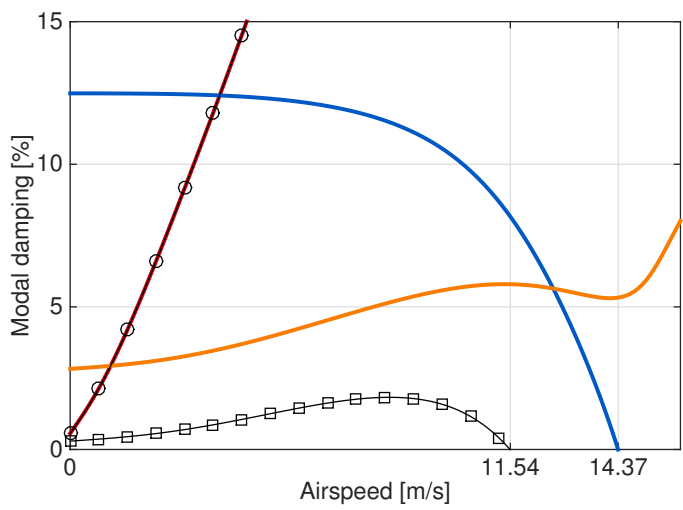

(f) Modal damping with $f_{l t v a}=2.0 H z, \zeta_{l t v a}=14.47 \%$

Figure 11: Effect of the LTVA on the system modal damping and frequency variation with airspeed 


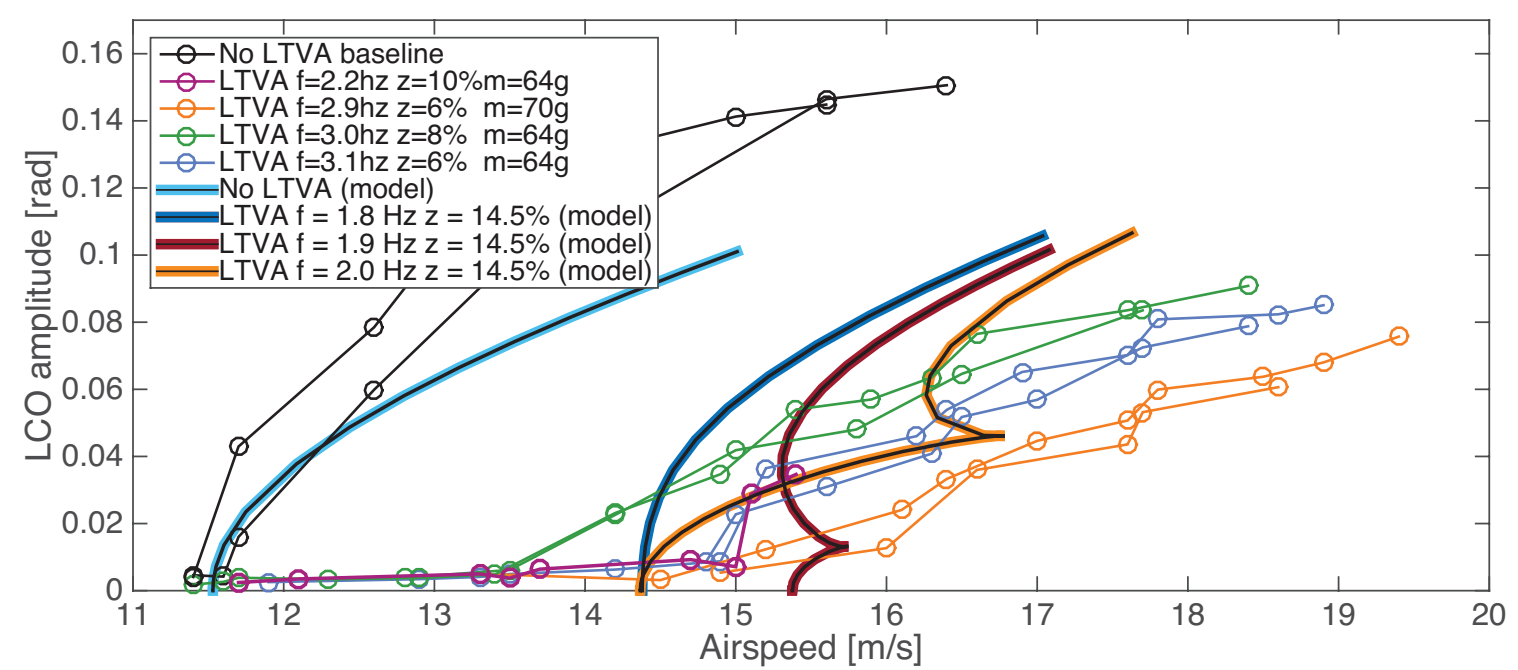

(a) Pitch amplitude bifurcation diagram

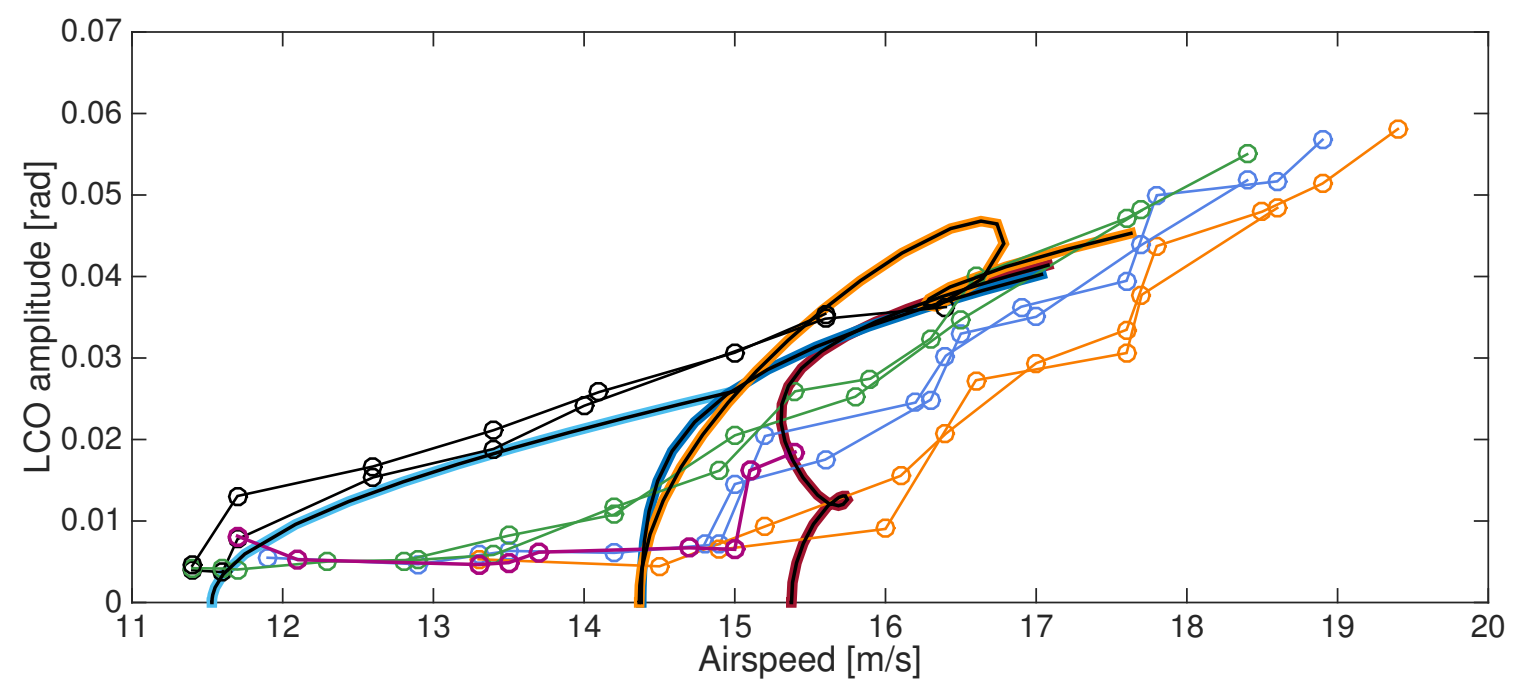

(b) Flap amplitude bifurcation diagram

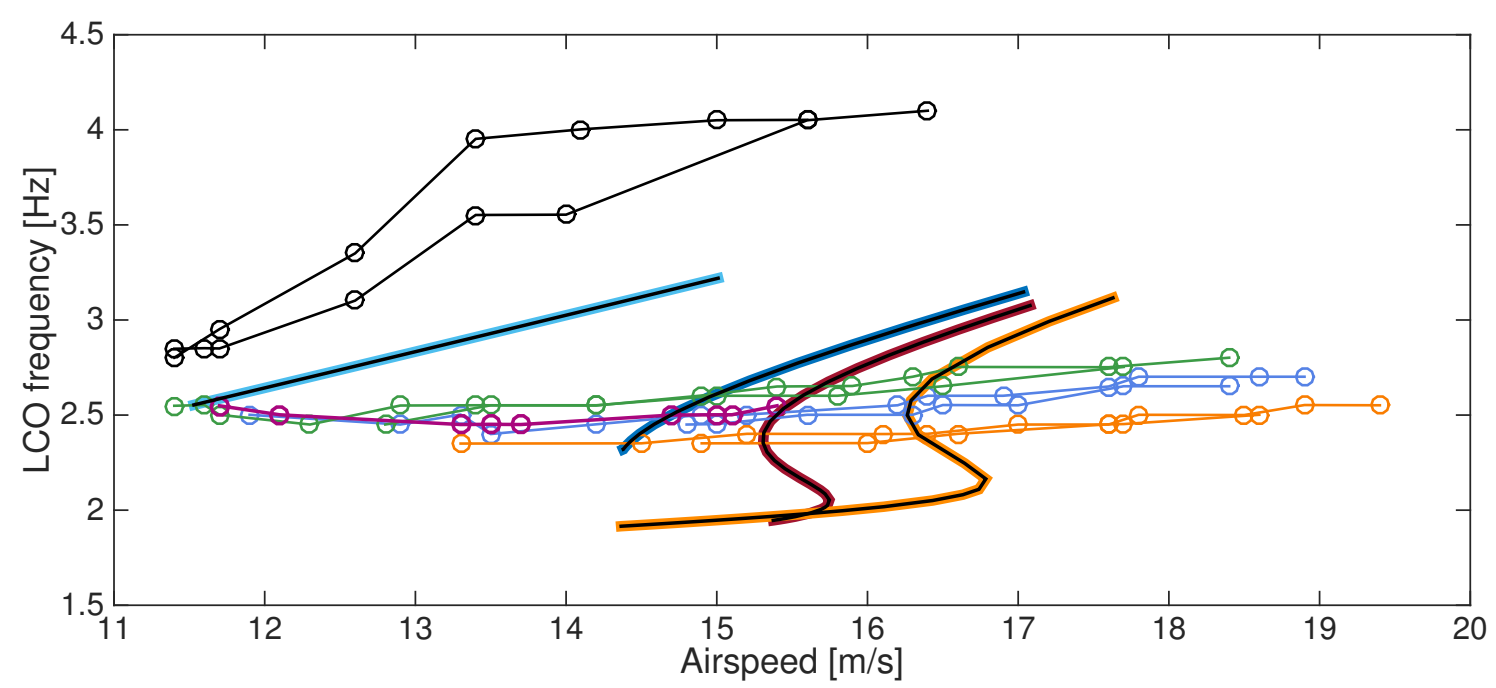

(c) LCO frequency bifurcation diagram 